\title{
Iris Nevus Disease Diagnosis using Convolutional Neural Network based on SURF (Speeded up Robust Feature) Detection
}

\author{
O.O Obe, $\mathrm{PhD}$ \\ School of computing, Computer Science \\ Department, \\ Federal University of Technology, \\ Akure, Nigeria
}

\author{
Olotuah Adedolapo Fisayo \\ School of computing, Computer Science \\ Department, \\ Federal University of Technology, \\ Akure, Nigeria
}

\begin{abstract}
This work presents the diagnosis of iris nevus (Cogan Reese) using a convolutional neural network $(\mathrm{CNN})$ for its classification and Speeded Up robust feature (SURF) detection for its feature extraction. Iris nevus is a tumor found in the eye. Racial and environmental factors affect the color of the iris of a patient; hence, tumor may be seen in the eye background. In this work, the iris images will be tested and trained and will also describe the automatic diagnosis of iris nevus using neural network-based systems for its classification as nevus affected and unaffected iris. The model attained its best performance during training and testing with an accuracy of $97.50 \%$ and $80 \%$ respectively, a precision of $77 \%$ and a recall of $67 \%$.
\end{abstract}

\section{Keywords}

CNN, SURF, Iris Nevus

\section{INTRODUCTION}

Inventors have dreamt of developing machines that think like human. This desire dated back to the ancient Greece. Talos, and Pandora may all be regarded as artificial life (Ovid and Martin 2004, [5] Sparkes 1996, [6] Tandy 1997 [7]. When programmable computers were first developed, people wondered if such machines might become intelligent, (Lovelace 1842) [2]. Today, artificial intelligence (AI) is a thriving with many practical applications and research purpose. The difficulties faced by systems relying on coded knowledge suggest that artificial intelligent systems need the ability so as to acquire their own knowledge, by extracting patterns from raw data. This capability is known as machine learning. The introduction of machine learning (ML) allowed computers to solve problems which involves knowledge of the real world and decision making

Deep learning solves this problem in representing learning by introducing representations that are expressed in terms of other, simpler representations. Deep learning allows computer to build complex concepts out of simpler concepts. The example of a deep learning model is the feedforward deep network or multilayer perceptron (MLP). A multilayer perceptron is a mathematical function that maps some set of input values to output values. Iris nevus is a tumor found in the background of the eyes. It has been proved by medical experts that the tumor is capable of transforming into iris melanoma which is malignant and can requires critical medical attention. The transformation of iris nevus into melanoma normally occurs at the ages of 1 to 20years

\subsection{The Contributions of this Research Work are as follows \\ (i) Design an iris nevus predictive model using convolutional neural network $(\mathrm{CNN})$; and \\ (ii) Implement and evaluate the model in (i)}

\section{RELATED WORK} INTELLIGENT DECISION SUPPORT SYSTEMS TO MODEL THE DIAGNOSIS OF IRIS NEVUS

Oyedotun et. al (2013) [3], This research work proposed the use of intelligent decision support systems which model the diagnosis of iris nevus. The systems used in this work include support vector machines (SVM), back propagation neural networks, and radial basis function networks. In this research, eye images of patients were collected. These images were processed so as to extract and reinforce features and regions of interest (ROI), respectively. The already processed images were used to train the designed networks. Support vector machine implemented with kernel shows the highest performance with a recognition rate of $90.8 \%$, while a recognition rate of $72.5 \%$ was obtained from the other support vector machine model implemented with a linear kernel.

\subsection{Iris Nevus Disease Diagnosis System using CNN and Deep Belief Network}

Oyebade et al 2017 [4] developed a system that diagnose iris nevus disease using convolutional neural network (CNN) and deep belief network (DBN). The two are biologically inspired neural networks for learning the classification of processed eye images into nevus-affected and unaffected categories. During the training phase of the neural networks, it was observed that DBN took less time and less iteration for training compared to the CNN. Effective image filtering was not carried out while classifying with CNN. At the end of this research, a system that diagnoses iris nevus disease using $\mathrm{CNN}$ and DBN was achieved.

\subsection{Coronary Heart Disease Diagnosis System using Deep Neural Networks}

Kathleen et al 2018 [1] designed a system that diagnoses coronary heart diseases using Deep Neural Networks architecture. In this work, a classification and prediction model was carried out based on a deep learning algorithm. The DNN model was used in diagnosing coronary heart disease and 303 clinical datasets was applied. The model was trained and tested. The performances of the developed DNN model was evaluated using diagnostic accuracy, specificity, probability of misclassification error, precision, sensitivity, F-score, and K-S test. 


\section{METHODOLOGY}

This chapter presents the architecture of the developed system with detailed analysis and algorithms of each system component and subcomponents.

The proposed system is composed of four major modules, the first module is the training and testing of the data collected, the second module has to deal with filtering of the image dataset using SURF feature detection, the images are passed to the pooling layer where sub-sampling of the images are carried out and the images are passed to the fully connected layer. Classification of the images is done in the third module using sigmoid function, while classification result and evaluation is carried out in the last module. The conceptual diagram of the system is shown in figure 3

\subsection{Data collection component}

The data collection component holds images of nevus samples to be used. The data contain images of iris nevus and non-infected iris samples. The data is downloaded from the repository of the Eye Cancer Foundation with the followinglink: http://www.eyecancer.com/research/image-gallery/11/iris-

tumors. The dataset contains a total of 403 samples with 148 samples labeled as iris nevus infected samples and 255 samples as normal samples. Figure 1 and Figure 2 show samples of infected and non-infected samples respectively.

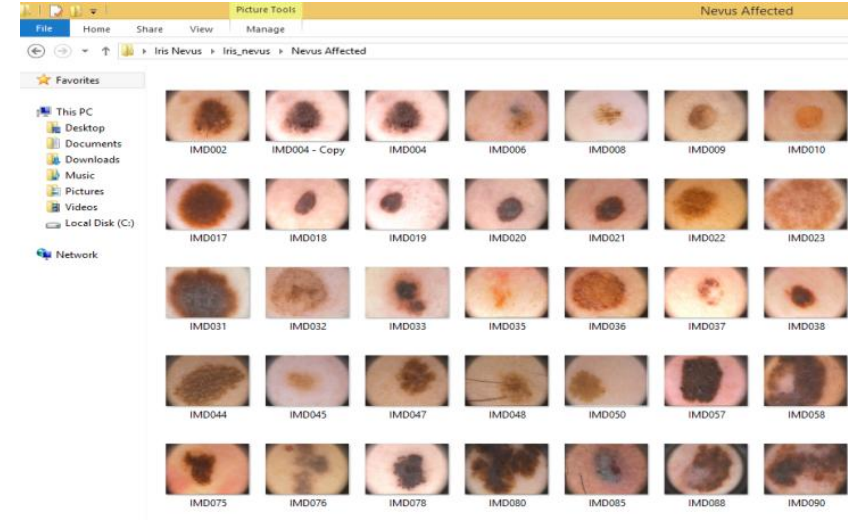

Figure 1: Image Dataset of Affected Iris

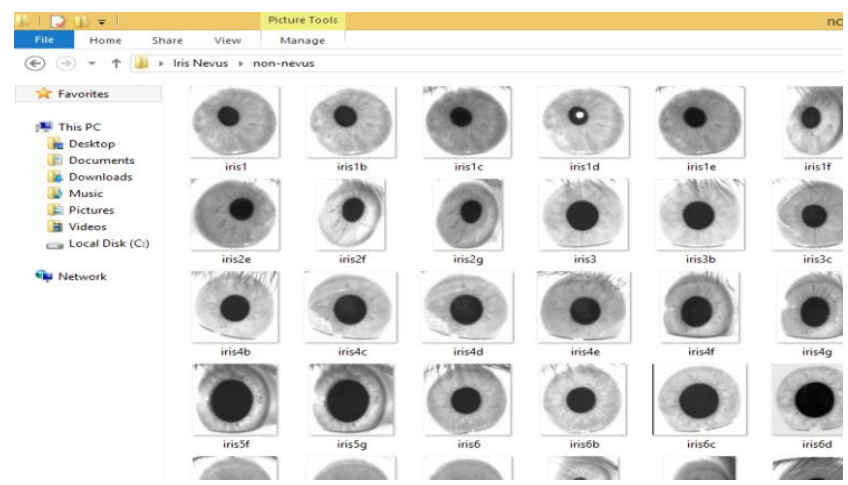

Figure 2: Image Dataset of Non- Nevus Iris

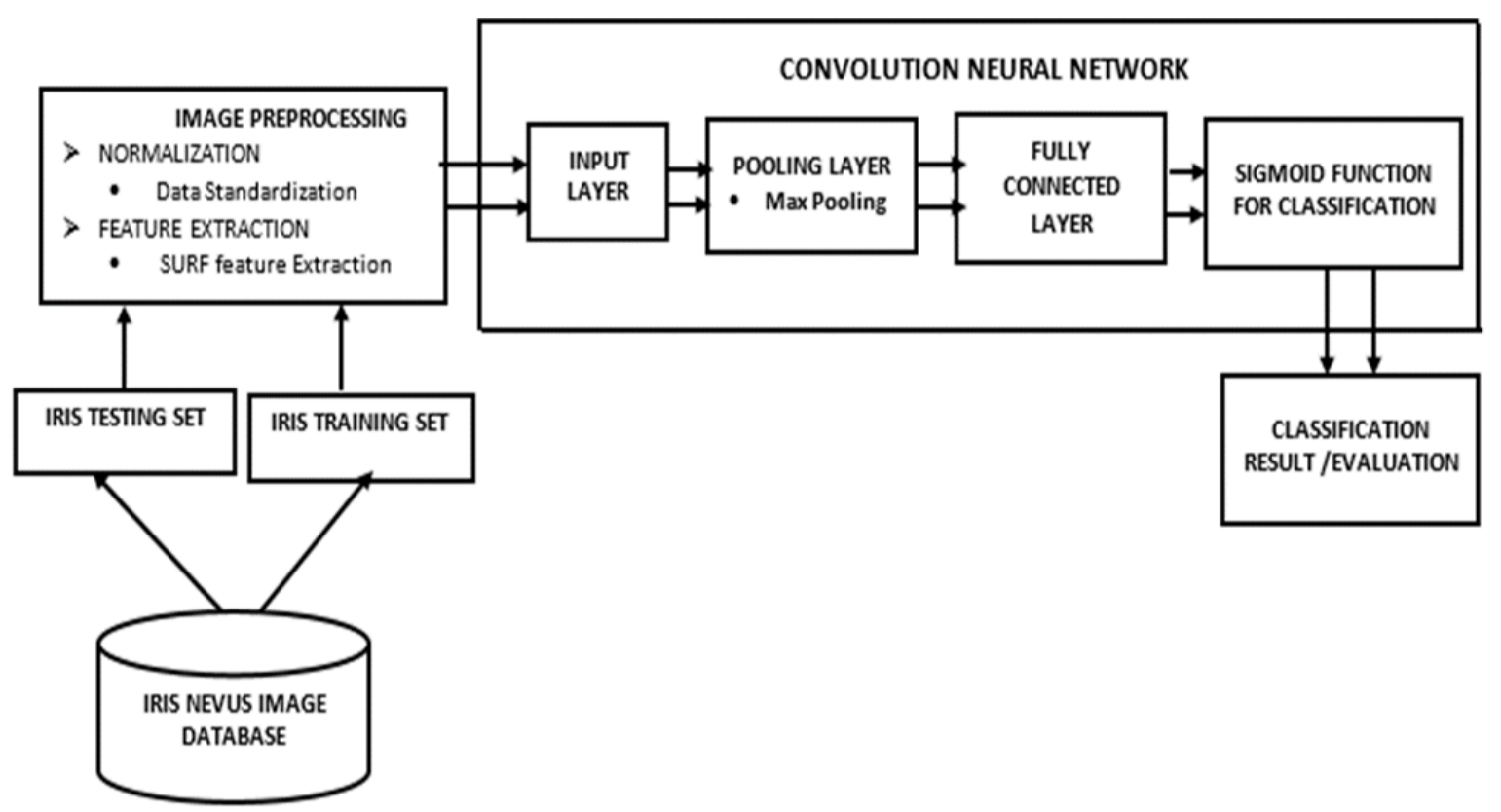

Fig 3: Architectural Design of the System

\subsection{Image Preprocessing Component}

The preprocessing component ensures that the image samples were prepared and made suitable for classification. The preprocessing steps used in this research include:

- Data Normalization: This process is applied to bring the image samples into a range of intensity values that is normal as well as to avoid the influence of high frequency and low noise in the course of classification. In this research, the standardization method was adopted as presented in equation (1)

$$
E I_{\text {new }}=\frac{\left(I_{v}-I_{\text {mean }}\right)}{I_{\text {std }}}
$$

where $I_{\text {new }}$ represents the pixel value of am image, $I_{v}$ represents the image value to be normalized, $I_{\text {mean }}$ represents the mean values obtained from the images, and $I_{\text {std }}$ is the standard deviation value of all the images. 


\subsubsection{SURF feature detection. (Speeded Up Robust Features) Algorithm}

Feature detection is process where we examine an image to extract features that are unique in the image.

This detection is possible when the image shows their transformations or when parts of the object are occluded. The processes can be divided in to 2 possible steps. Which are Detection and Description

\subsubsection{Feature Extraction}

The feature extraction component ensure the relevant image features were used for the purpose of classification. This is to ensure improved classification performance and reduced computational time. This research adopted SURF (Speeded Up Robust Feature) extraction technique for this purpose.

- $\quad$ SURF is a local feature descriptor designed to improve the limitation encountered in SIFT (Scale Invariant Features Transform).

- It is fast and can accurately detect key features of images for better classification performance at reduced time cost.

In this research, Surf uses Hessian matrix approximation to detect interest points and the sum of Haar wavelength responses to identify and extract best descriptors from images using the following equations:

Given that image $u$ as a processed digital image defined over the pixel grid $\Omega$, the integral image of $u$ for image point $(x, y) \in \Omega$ given as:

$$
U(x, y)=\sum_{0 \leq i<x} \sum_{0 \leq j \leq y} u(i, j)
$$

where $U(x, y)$ is the integral image. The integral image is computed so as to achieve robustness to color modifications.

Once the image has been computed, two consecutive steps are performed to determine the relevant features as follows:

\section{STEP 1}

- $\quad$ Feature filtering: This detects interesting features or points in the box-space that are highly discriminants. Interest points are defined as the local maxima of Determinant of Hessian at scale $\mathrm{L}\left(\mathrm{DoH}^{\mathrm{L}}\right)$ operator applied to the image $\mathrm{u}$ as expressed in equation (3)

$$
\left.D o H^{L}(\mathrm{u})=\frac{1}{L^{4}}\left(D_{x}^{L} \mathrm{u}^{*} D_{y y}^{L} \mathrm{u}\right)-\left(\mathrm{w} D_{x y}^{L}\right)^{2}\right)
$$

where $D o H^{L}(\mathrm{u})$ represents the determinant of Hessian, $\frac{1}{L^{4}}$ is the normalization factor to ensure scaling invariance as $\mathrm{L}=2^{\circ} i+1$, $\mathrm{w}$ is the weighting factor set as $0.912, D_{x}^{L}$ represent the first order box filter, $D_{y y}^{L}$ and $D_{x y}^{L}$ represents the second order point filters at scale $\mathrm{L}$.

\section{STEP 2}

- Feature selection: The maxima are detected and selected by considering a $3 \times 3 \times 3$ neighbourhood, and performing an with its 26 nearest-neighbours.

\subsection{Classification Component}

At this stage, the proposed system adopted Convolutional Neural Network (CNN) for the classification Nevus and Non-nevus iris images. CNN is composed of 3 basic layers called:

\subsubsection{Convolutional layer (Input- layer)}

The convolutional- layer accepts extracted feature vectors presented in resized shape $100 * 100$ dimension with channel depth 3 from the preprocessing components. At this layer, each feature from an image is represented by feature map, and the feature map is the outcome of every filter that convolved through the entire image as described in equation (4)

$x_{j}^{l}=\mathrm{f}\left(\sum_{i \in M_{j}} x_{i}^{l-1} * k_{i j}^{l}+b_{j}^{l}\right)$

where $j$ is the specific feature map, $M_{j}$ as the selection of the input maps, $k_{i j}$ is the filter, $b_{j}$ is the feature map bias, $l$ is the layer in the $\mathrm{CNN}$, and $f$ is the activation function called ReLU

\subsubsection{Pooling layer}

Pooling layers are generally used to reduce the size of the inputs so as to speed up the computation. The pooling operation is given as follows:

$x_{i}^{l}=f\left(B_{i}^{l} \operatorname{maxpool}\left(x_{j}^{l-1}\right)+b_{i}^{l}\right)$

where $x_{i}^{l}$ is the result of the down sampling operation applied on the $j^{\text {th }}$ region of the input, $x_{j}^{l-1}$ is the $j^{\text {th }}$ region of interest captured by the maxpooling task, $B_{i}^{l}$ is the trainable coefficient, and $b_{i}^{l}$ is the trainable bias.

\subsubsection{Fully connected layer}

At this stage, the result of the pooling layer is fed into the fully connected layer for classification. This layer contains the sigmoid function that classified the images data as iris nevus disease or a normal data using equation (6)

$y=\operatorname{sigmoid}\left(w_{o} x_{i}^{l}+b_{o}\right)$

Where y represents the output (class label of either normal or nevus), $w_{o}$ represents the output weight, and $b_{o}$ represent the output bias.

\section{EVALUATION}

This section shows the graphical representations of training and test accuracy and loss on images with and without features detection.

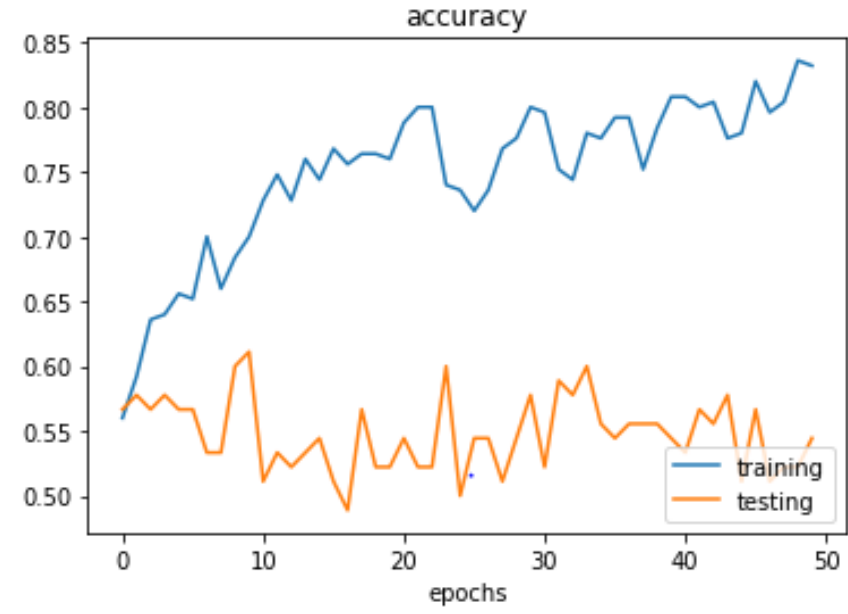

Figure 4: Graphical representation of training and test accuracy on images without features detection

The observation in figure 4 shows an overfitting as the predictive model performed too well on the training data but produces an average result of $61.1 \%$ when fed with test data. It is observed that actually learnt the detail and noise in the training data till it negatively impacts the performance of the model on test data. 


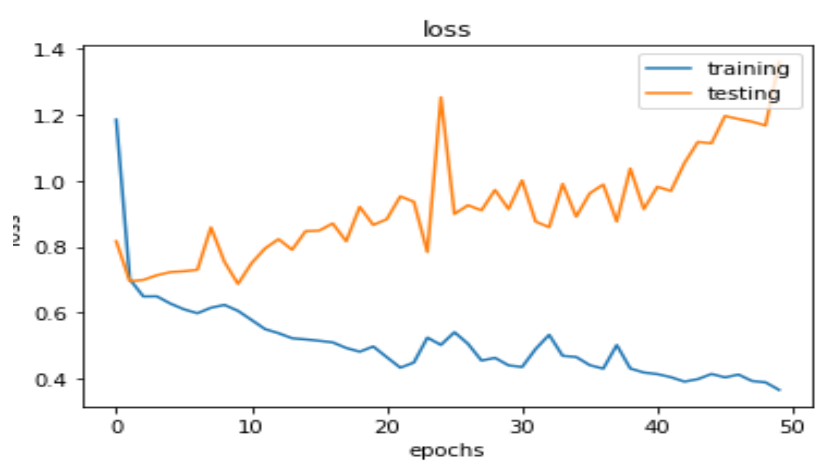

Figure 5: Graphical representation of the loss in training and test on images without features detection

It is observed figure 5 that there was not significant loss during the testing phase as the graph shows a constant rise in loss rate from epoch 7 .

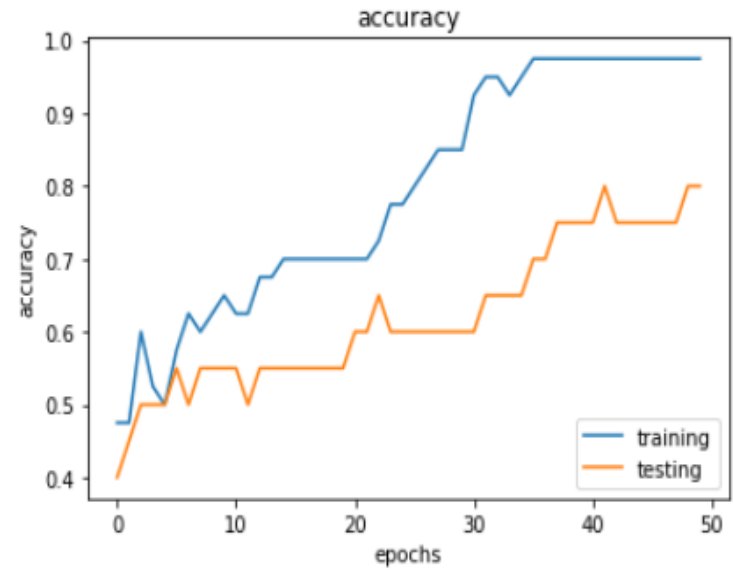

Figure 6: Graphical representation of training and test accuracy on images with features extraction

The observation in figure 6 indicates that there was gradual increase in the model's prediction on training and test data as the number of epoch increases. Here, the model attained its best performance during training and testing at the $36^{\text {th }}$ and 42th epoch with an accuracy of $97.50 \%$ and $80 \%$ respectively.

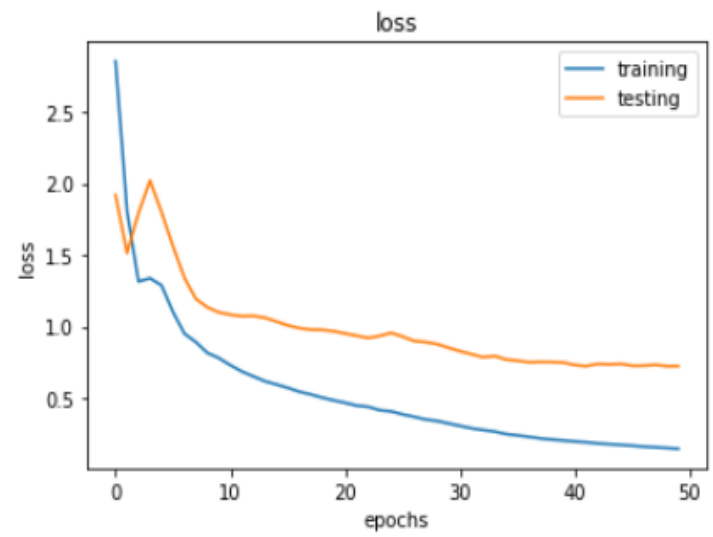

Figure 7: Graphical representation of the loss in training and test on images with features extraction
The loss shown in figure 7 indicated model's ability to minimize loss during gradient decent as it decreases with increase in the number of epoch

\subsection{RESULTS}

This module displays classification results as well as the report of the system's performance. The metrics adopted for performance evaluation is also used for the systems. Evaluation such as Recall, and Precision, Accuracy (ACC). Based on confusion matrix, the equation of the metrics is as follows

The performance evaluation of the proposed model will be carried out based on the following metrics and is presented as follows:

\subsubsection{Accuracy:

$$
A C C=\frac{T P+T N}{T P+T N+F P+F N}
$$

where (TP) which is true positive represents data detected, (TN) which is True Negative represents normal data detected as normal, (FP) which is False positive represents normal data detected, and (FN) which is False Negative is denoted as data detected as normal

\subsubsection{Precision}

Basically tells us that out of the results classified as positive by our model.

Precision $=\frac{\text { true positive }}{\text { true positive }+ \text { false positives }}$

\subsubsection{Recall}

Tells us how many true positive were found by the model. It is the number of items correctly identified as positive out of the total items identified as positive.

Recall $=\frac{\text { true positive }}{\text { true positive }+ \text { false negatives }}$

Below is The confusion-matrix table on test data without surf features and with surf feature detection respectively

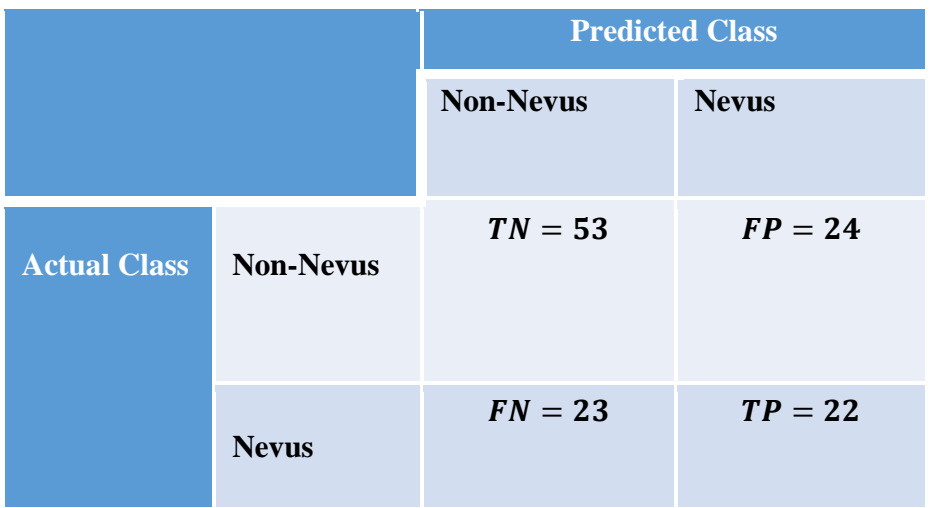

Precision $=T P /(T P+F P)^{*} 100$

$=22 /(22+24)=22 / 46=0.48 * 100=48.0 \%$

Recall $=T P /(T P+F N)=22 /(22+23)=0.49 * 100=49.0 \%$ 

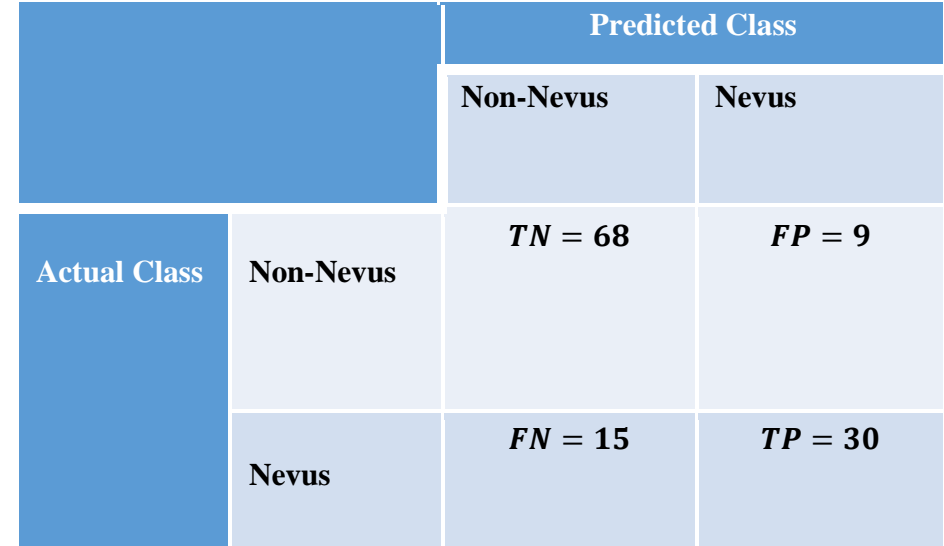

Precision $=T P /(T P+F P) * 100$

$=30 /(30+9)=30 / 39=0.77 * 100=77.0 \%$

Recall $=T P /(T P+F N)=30 /(30+15)=0.67 * 100=67.0 \%$

In table 1, it was observed that we got a Precision of $48 \%$ and a Recall of $49 \%$ when the data was tested without feature extraction and in table 2, it shows that the Precision and recall has increased to $77 \%$ and $67 \%$ respectively.

The confusion- matrix table shows True positive, True Negative, False Positive, and False Negative of CNN nevus predictive model on test data with SURF selected features. The table shows that the model correctly classified 98 image data from 122 test images. This was obtained by adding the value of $\mathrm{TP}$ and TN:

Correct Classification $=\mathrm{TP}+\mathrm{TN}=30+68=98$

Incorrect Classification $=\mathrm{FP}+\mathrm{FN}=9+15=24$

\section{CONCLUSION AND FUTURE WORK}

CNNs are a powerful technique that we experiment with in this research. SURF (Speeded up robust feature) was used for feature detection of the iris. The technique is very easy in comparison with the other techniques. It does not need any information about pupil to discover location of the iris. Consequently, it does not need guess potential iris centers and Radii. So, the process not consume a lot of time, and complexity of computations. At the same time, it gave comparable performance like the other techniques.

In this research work, we described a SURF based convolutional neural network (CNN), for classifying of iris nevus disease which is the inspired neural networks architecture for learning the classification of processed eye images into nevus-affected and non-affected categories. During the training phase of the network when the data were fed directly into the model, the accuracy shows the performance of the model on train and validation set. The validation set shows the inability model to generalize well on new nevus eye data and also shows that model keeps improving while the accuracy is on average.

During the training and test on the data without feature extraction, it is observed that there was not significant loss during the testing phase as the result shows a constant rise in loss rate from epoch 7 .
During the training and test on the data with feature extraction observation that there was gradual increase in model's accuracy on training and test data as the number of epoch increases. The model attained its best performance during training and testing at the 36th and 42th epoch with an accuracy of $97.50 \%$ and $80 \%$ respectively.

The loss indicated model's ability to minimize loss during gradient decent as it decreases with increase in the number of epoch. Several future possibilities are identified for getting better performance with CNNs. It is noted that pooling layers can be changed in such a way to obtain benefits from max pooling without pushing the networks to over fit.

At the end of this research, I consider this implementation a successful one. This is able to filter iris images using SURF algorithm and to classify the images into nevus affected and non-nevus affected using Convolution Neural Network (CNN) with consistent results which will work significantly in the detection of iris nevus greatly to reduce the risk of iris nevus transformation into iris melanoma and secondary glaucoma.

\section{ACKNOWLEDGMENTS}

I would like to thank my supervisor, Dr. O.O Obe for his wise guidance during my studies and research. This thesis would not have been possible without the intelligent guidance. I also would like to thank the entire staff of school of computing both academic staff and non-academic for their time and willingness to offer advice and guide me in my studies.

\section{REFERENCES}

[1] Kathleen. S, 2018: Development of coronary heart diagnosis system using Deep Neural Network.

[2] Lovelace, A. (1842). Notes upon L. F. Menabrea's “ Sketch of the Analytical Engine invented by Charles Babbage

[3] Oyedotun O K, Olaniyi E O, Helwan A, Khashman A. Decision support models for iris nevus diagnosis considering potential malignancy. International Journal of Scientist

[4] Oyedotun O K, Olaniyi E O, Khashman, 2015. A Deep learning in character recognition considering pattern invariance constraints. International Journal of Intelligent Systems and Applications; 1-10.

[5] Ovid and Martin, C. (2004). Metamorphoses.

[6] Sparkes, B. (1996). The Red and the Black: Studies in Greek Pottery. Routledge.

[7] Tandy, D. W. (1997). Works and Days: A Translation and Commentary for the Social Sciences. University of California Press.

[8] Wikipedia, "Feature detection - Wikipedia, the free encyclopedia," 2011, [Online; accessed 14-July-2010]. [Online].

Available: https://secure.wikimedia.org/wikipedia/ detection \%28computer vision $\% 29$ 